\title{
ARTICLE OPEN \\ Distribution of metallic fission-product particles in the cladding liner of spent nuclear fuel
}

Richard A. Clark (iD ${ }^{1,4 *}$, Michele A. Conroy $\mathbb{D D}^{1,2,4}$, Timothy G. Lach $\mathbb{D}^{1}$, Edgar C. Buck $\mathbb{D}^{1}$, Kristi L. Pellegrini ${ }^{1,3}$, Bruce K. McNamara ${ }^{1}$ and Jon M. Schwantes (iD)

We have made observations of noble metal phase fission-product agglomerates and gaseous xenon within the fuel-cladding interaction $\left(\mathrm{FCl}\right.$ ) zone of a high-burnup $\mathrm{UO}_{2}$ fuel. The $\mathrm{FCl}$ is the boundary between the $\mathrm{UO}_{2}$ pellet outer surface and the inner wall of the oxidized $\mathrm{Zr}$-liner/cladding of the fuel rod. These fission-product agglomerates are well known to occur within the spent fuel matrix, and although radionuclides have been reported by others, we reveal aspects of their speciation and morphology. That they occur as discrete particles in the oxidized $\mathrm{Zr}$ liner, suggests the occurrence of hitherto unknown processes in the $\mathrm{FCl}$ zone during reactor operation, and this may have implications for the long-term storage and disposal of these types of materials. As expected, the particle agglomerates, which ranged in size from the nanometer scale to the micrometer scale, contained mainly Mo, Ru, Tc, Rh, and Pd; however, we also found significant quantities of Te associated with Pd. Indeed, we found nanometer scale separation of the distinct $\mathrm{Pd} / \mathrm{Te}$ phase from the other fission products within the particles. Often associated with the particles was concentrations of uranium, sometimes appearing as a "cloud" with a tail emanating from the fuel into the oxidized cladding liner. Many of the noble metal phase particles appeared as fractured clusters separated by Xe-gas-filled voids. Possible mechanisms of formation or transport in the cladding liner are presented.

npj Materials Degradation (2020)4:4 ; https://doi.org/10.1038/s41529-019-0107-0

\section{INTRODUCTION}

The fuel from boiling water reactors (BWRs) consists of uranium oxide $\left(\mathrm{UO}_{2}\right)$ ceramic pellets contained within a zirconium "barrier" liner that is in turn surrounded by Zircaloy cladding. The "barrier" consists of an inner layer of pure zirconium metal designed to curtail the transport of the fission-product iodine which can induce stress corrosion cracking (SCC) in Zircaloy cladding. The zirconium metal liner has become the preferred solution for BWR fuel manufacturers ${ }^{1}$ to prevent SCC. The liner, which becomes oxidized during reactor operation, prevents oxidation of the Zircaloy cladding layer and also provides impact resistance from fission fragments and fuel swelling. ${ }^{2}$ Prior to reactor operation, there is a gap filled with pressurized helium gas between the fuel and the liner. During operation, this gap closes as the fuel expands and makes contact with the $\mathrm{Zr}$ liner. Spent nuclear fuel (SNF) undergoes significant chemical and microstructural changes during reactor operations. ${ }^{3-8}$ Some fission products are retained within the $\mathrm{UO}_{2}$ matrix in solid solution, while at the same time the noble gases-xenon ( $\mathrm{Xe}$ ) and krypton $(\mathrm{Kr})$-and the $4 \mathrm{~d}$ group metals-molybdenum (Mo), technetium $(\mathrm{Tc})$, ruthenium $(\mathrm{Ru})$, rhodium (Rh), and palladium ( $\mathrm{Pd}$ ) - are trapped as gas bubbles and partitioned into metallic phases, respectively. ${ }^{9-14}$ This metallic phase has gone by several names in the literature, including white inclusions ${ }^{15,16}$ fission-product alloy, ${ }^{16} 5$-metal particles, ${ }^{17}$ epsilon particles, $^{18,19}$ and noble metal phase. ${ }^{6,20}$

Knowledge of the distribution of radionuclides across the fuel matrix or partitioning as discrete phases within or outside the fuel grains is necessary to make predictions about potential release in the event of cladding failure during SNF storage, transportation, or long-term geologic disposal. ${ }^{21-25}$
Changes to the $\mathrm{FCl}$ zone during reactor operation may also have a deleterious impact on the thermomechanical properties of the fuel. ${ }^{26}$ Ciszak et al., using micro-Raman spectroscopy to examine cross-sections through the $\mathrm{FCl}$ from high-burnup (HBU) pressurized water reactor (PWR) fuels, ${ }^{27}$ reported on various $\mathrm{Zr}$ oxide phases that form in this corrosion rind, speculating their existence may have resulted from phase transitions due to fission-product radiation induced defects or incorporation of contaminants into the lattice structure. Lach et al. went further; they used scanning transmission electron microscopy (STEM) to show that not only did the Zr-oxide phase depend on the degree of fission-product radiation with the high temperature tetragonal phase being stabilized, but also the grain size of the Zr-oxide strongly correlated with fission-product radiation. ${ }^{28}$

It has been known for some time that fission products can migrate to the cladding. Indeed, the distribution of radionuclides in cladding has been examined by Hirabayashi et al. using a stepwise etching process. It was found that $98 \%$ of fission products in the cladding were detected within a $10 \mu \mathrm{m}$ depth from the inner surface of the cladding. ${ }^{29}$ Here, a detailed microscopic analysis of the fission products in the cladding is presented which provide new insights to their formation and migration behavior.

Initial characterization of Approved Testing Material (ATM)-109, a SNF from a BWR from the Quad Cities I reactor (Quad Cities Generating Station, Cordova, Illinois, USA) was performed by Vaidyanathan et al. ${ }^{30}$ and Wolf et al. ${ }^{31}$ This fuel was irradiated from February 1979 to September 1987, and again from November 1989 to September 1992. The $\mathrm{UO}_{2}$ had an initial $3.0 \%{ }^{235} \mathrm{U}$ enrichment and an initial grain size of $\sim 30 \mu \mathrm{m}$. The specimens received a maximum burnup of $70-80 \mathrm{GWd} / \mathrm{MTU}$. The HBU resulted in more ${ }^{239} \mathrm{Pu}$ fission producing higher levels of $\mathrm{Pd}$ and

\footnotetext{
${ }^{1}$ Pacific Northwest National Laboratory, 902 Battelle Blvd, Richland, WA 99352, USA; ${ }^{2}$ Present address: Department of Physics, Bernal Institute, University of Limerick, Limerick V94 T9PX, Ireland. ${ }^{3}$ Present address: IB3 Global Solutions, Oak Ridge, TN 37830, USA. ${ }^{4}$ These authors contributed equally: Richard A. Clark, Michele A. Conroy. ${ }^{*}$ email: richard. clark@pnnl.gov
} 
Ag than would be present in lower burnup fuels. The $4 \mathrm{~d}$ group metals tend to form fcc metals rather than $h c p$ that is observed with Ru-Mo rich phases in nuclear fuels. ${ }^{32}$ We have previously reported on the nature of the metallic particles from these fuels and aspects of their chemistry, including the variability of compositions in the 5-metal particles and the occurrence of Ag-Pd halides. ${ }^{13,33-36}$ In these earlier studies, we investigated metallic particles throughout the fuel; however, in this investigation, we concentrated on the $\mathrm{FCl}$ region and, in particular, the oxidized Zr metal liner nearest to the fuel.

\section{RESULTS}

Analysis of noble metal phase particles in cladding liner

A cross-sectional montage of ATM-109 is shown in Fig. 1a. The cladding including the zirconium metal and corrosion rind was $851 \pm 6 \mu \mathrm{m}$ thick with an outer corrosion layer $30.0 \pm 3.3 \mu \mathrm{m}$ thick based on scanning electron microscopy (SEM) measurements. Hydrides were visible in the Zircaloy with an increased concentration of hydrides at the interface between the Zircaloy and zirconium liner (see Supplementary Fig. 1, Supporting Information $(\mathrm{SI})$ ). The presence of the hydrides made it difficult to measure the exact thickness of the liner but this appeared to be $56 \pm 5 \mu \mathrm{m}$. Evidence for the extent of the fuel-cladding interaction $(\mathrm{FCl})$ is shown in Fig. 1b where an oxide layer has formed in the gap region and has contacted the expanding $\mathrm{UO}_{2}$ surface. The inner part of the zirconium liner had formed an oxide layer that was on average $10 \pm 2 \mu \mathrm{m}$ thick, although some areas were $15 \mu \mathrm{m}$ thick. The higher magnification image in Fig. 1c shows "jets" of uranium (bright contrast) released into the zirconium oxide layer. It is also noteworthy that the outer surface of the $\mathrm{UO}_{2}$ fuel was generally devoid of gas bubbles relative to the rest of the fuel and even relative to the oxidized $\mathrm{Zr}$ layer of the cladding. The existence of this interesting morphology is discussed in greater detail elsewhere (see Schwantes et al. ${ }^{37}$ ). The occurrence of $U$ in the oxidized zirconium layer was confirmed with energy dispersive $\mathrm{x}$-ray spectroscopy (EDS). Elemental maps of Fig. 1c for $U$ (yellow) and $\mathrm{Zr}$ (orange) are shown in Fig. 1d. Olander ${ }^{38}$ has estimated the oxidation of the $\mathrm{Zr}$ metal layer is caused by the release of oxygen from $\mathrm{UO}_{2}$ during fission and uptake of the oxygen by the $\mathrm{Zr}$ liner at high temperatures. The backscattered contrast from the oxidized zirconium appears slightly less bright in Fig. 1d than the $\mathrm{Zr}$ metal, owing to lower average atomic number of the zirconium oxide. Similar oxide layers have been observed in highburnup fuels by Walker et al. $^{39}$ and by Ciszak et al. ${ }^{27}$ and are discussed in detail for this fuel by Lach et al. ${ }^{28}$

Additional SEM-EDS spectral image maps of the oxidized liner separated from the fuel are shown in Fig. 2. Along with the presence of uranium, the five elements comprising the noble metal phase inclusions (Mo, Tc, Ru, Rh, and Pd) were identified in the layer. This demonstrated that the metallic particles were intact and contained within the $\mathrm{ZrO}_{2}$ layer. For the larger particles, there was often an elevated concentration of uranium surrounding the particle appearing as a "cloud" with a trail of uranium leading back to the cladding/fuel interface (Fig. 3 and large particle in lower right corner of Fig. 2 images). The "cloud" continued to be observed when lift-outs were obtained using a focused ion beam (FIB)-SEM for side-views (Fig. 4) and TEM analysis. Slice and view analyses also observed concentrations of uranium around the particles. The imaging invokes a dynamic or forceful intrusion of uranium into the $\mathrm{Zr}$ barrier. Similar images of the interaction of $\mathrm{UO}_{2}$ and the $\mathrm{Zr}$ layer have not been recorded prior to this work, although others ${ }^{40}$ have sited the alteration of the $\mathrm{Zr}$ barrier by $\mathrm{UO}_{2}$ in irradiated specimens of nuclear fuel. Though not observed in the EDS analysis with SEM, smaller particles also occasionally had slightly elevated uranium concentrations in the immediate vicinity when analyzed with STEM-EDS. Further, SEM-BSE imaging and EDS mapping throughout the liner-oxide confirmed there was a correlation between the uranium and many of the noble metal phase particle distributions with larger noble metal phase particles present in the areas of higher uranium concentration.
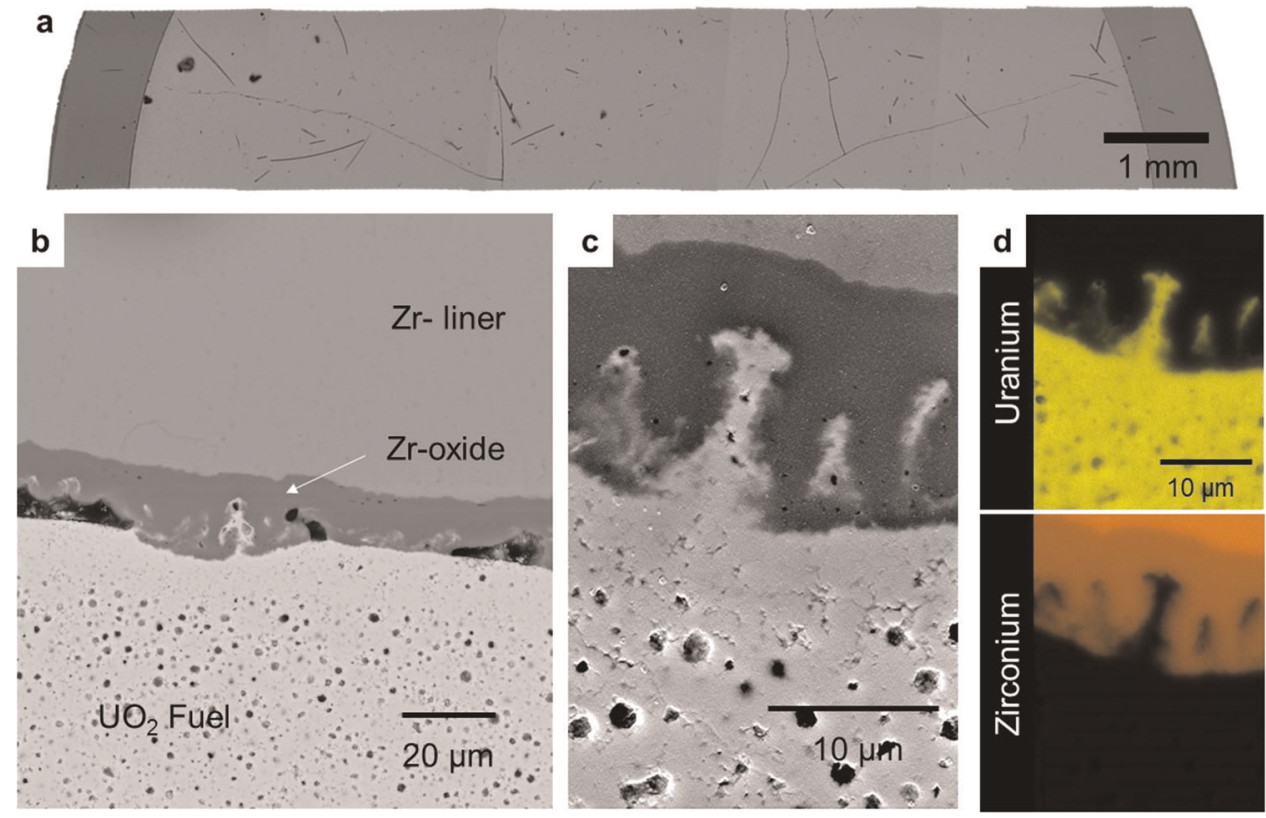

Fig. 1 Imaging the cross-section of spent fuel. (a) SEM-BSE images montaged across the entire spent fuel section showing the cladding and fuel thickness (see the SI for additional images (Supplementary Fig. 2) of the ATM-109 fuel). The few black lines on the micrograph of the fuel are artifacts from carbon coating the sample. (b) SEM image of the spent fuel/Z $\mathrm{rO}_{2} / \mathrm{Zr}$-liner interface region showing "jets" of uranium penetrating the $\mathrm{ZrO}_{2}$ layer. (c) Higher magnification of Fig. $1 \mathrm{~b}$ at the interface between the cladding liner and the fuel. Noble metal phase particles are observable and show up as bright specs in the image. (d) EDS spectral image mapping of the liner showing the contrast between $\mathrm{Zr}$ and regions of $\mathrm{U}$ intrusion into the FCl. Figure 1c reprinted from Journal of Nuclear Materials, 521, Lach, T. G. et al., Fission recoil-induced microstructural evolution of the fuel-cladding interface [FCI] in high burnup BWR fuel, 120-125, Copyright (2019), with permission from Elsevier. 

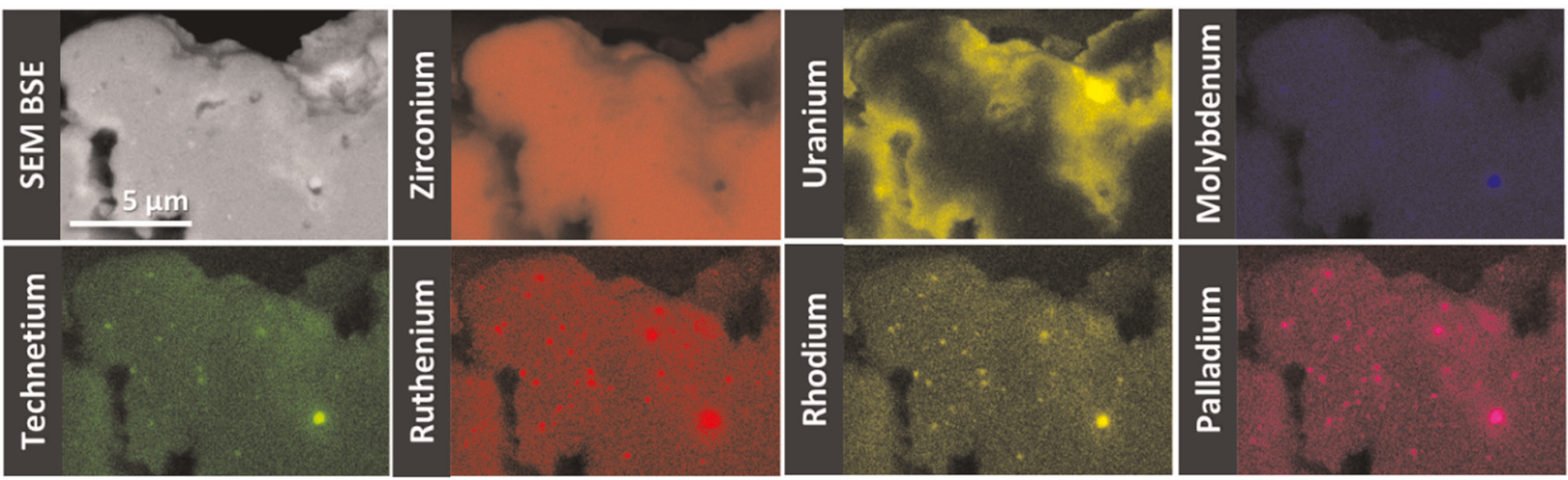

Fig. 2 Elemental maps of the Zr liner. SEM-BSE image and EDS spectral images of Zr, U, Mo, Tc, Ru, Rh, and Pd in the oxidized liner showing epsilon metal particles embedded in the material.

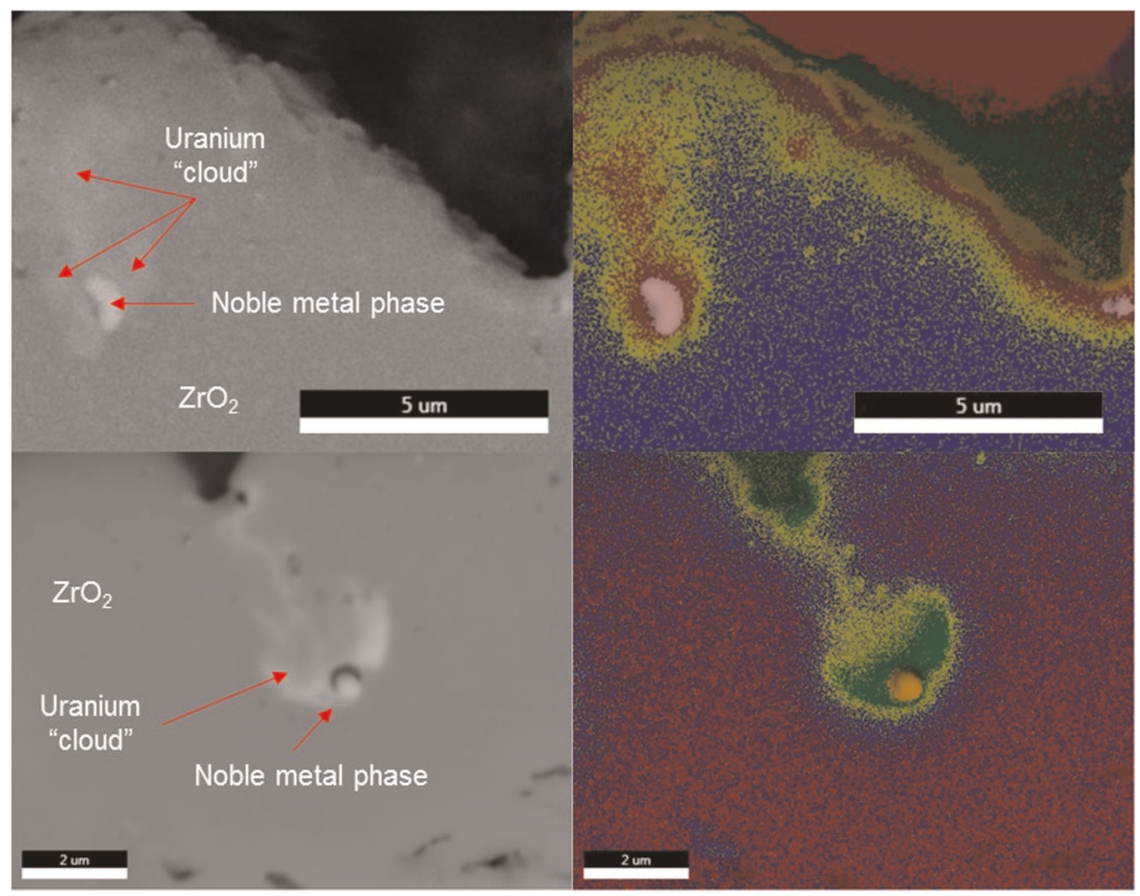

Fig. 3 Noble metal phase particles and uranium in the Zr liner. Images of noble metal phase particles surrounded by uranium "cloud" (higher (red-upper-right and green-lower-right) and lower (yellow) concentrations of $U$ for the images on right) with trail back to the fuel/ cladding interface. A few smaller metallic particles are also visible especially along the trail of uranium. Lower two panels reused from Schwantes, Jon M., et al. A new non-diffusional gas bubble production route in used nuclear fuel: implications for fission gas release, cladding corrosion, and next generation fuel design. Physical Chemistry Chemical Physics (2020) - Reproduced by permission of the PCCP Owner Societies.
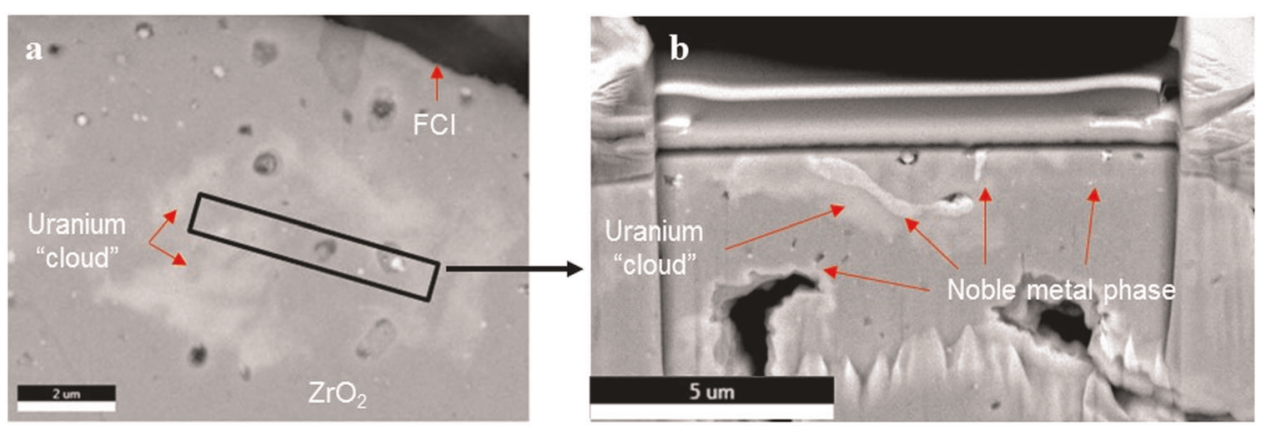

Fig. 4 Variations in size and shape of noble metal phase particles. (a) SEM-BSE image of noble metal phase particles in the cladding liner. (b) A slice removed from the cross-section using a FIB-SEM. A large distribution in size and shape of the noble metal phase particles is observed. The uranium "cloud" persists with the side-view. 

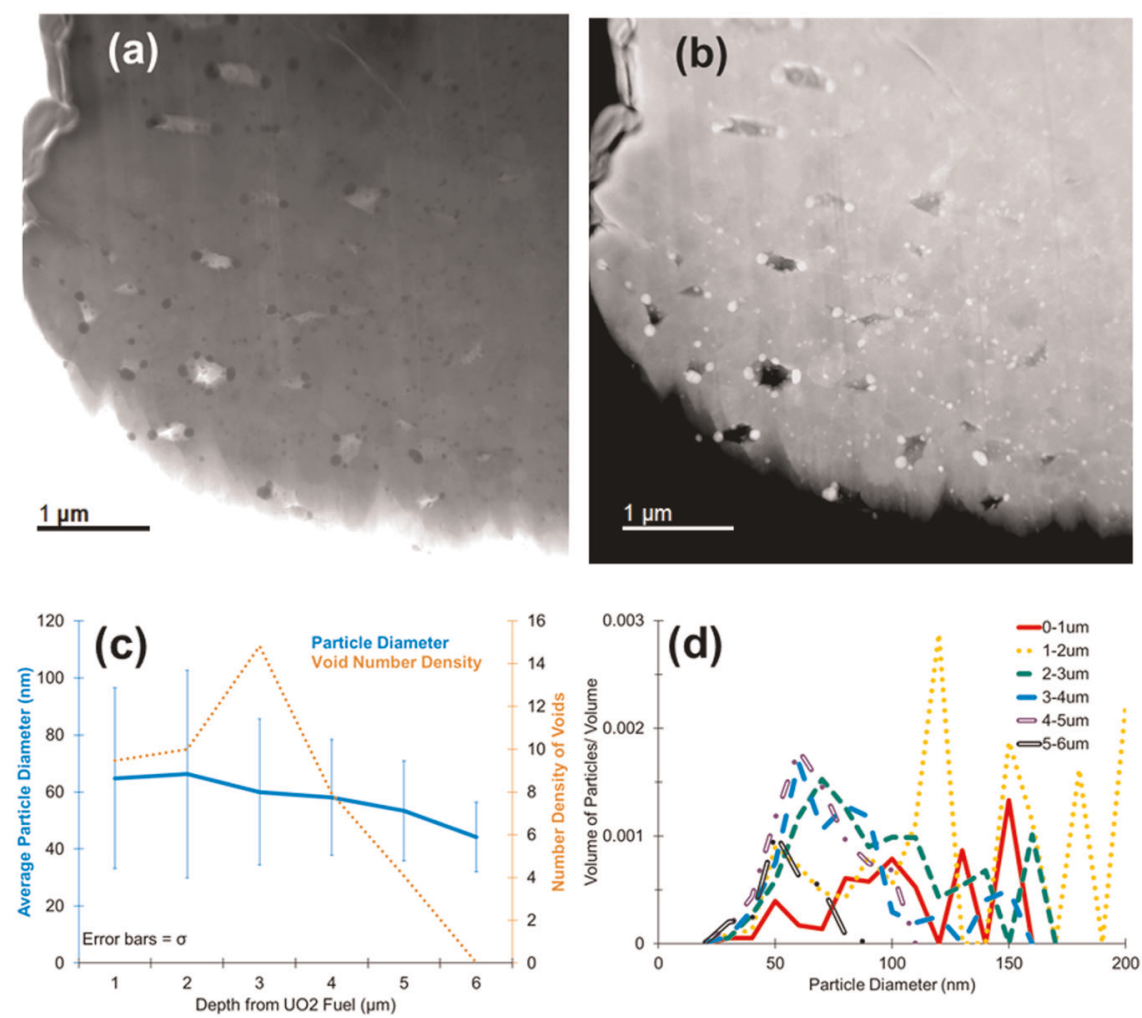

Fig. 5 Noble metal phase particles within the Zr liner. (a) STEM BF and (b) STEM HAADF images of the altered Zr oxide region nearest the $\mathrm{UO}_{2}$ fuel (left side of image) with particle morphology variation evident. (c) Average particle diameter and void number density as a function of depth into the $\mathrm{Zr}$ oxide from the $\mathrm{UO}_{2}$ fuel. (d) Particle volume density as a function of particle diameter for depths between 0 and $6 \mu \mathrm{m}$ depths from the fuel.

To study these features in greater detail, thin section foils normal to the interface between the fuel and cladding liner were prepared for scanning transmission electron microscopy (STEM)/ transmission electron microscopy (TEM) investigations using FIBbased lift-out techniques (see Fig. 5). The voids and particles nearest the fuel side (left side in Fig. 5) could be seen within $\sim 5-6 \mu \mathrm{m}$ of the fuel. Small noble metal particles were found in all samples taken near the fuel interface. The morphology and size distribution of the noble metal phase particles varied as a function of depth from the $\mathrm{UO}_{2}$ fuel into the altered Zr-oxide region, as seen in the STEM bright-field (BF) and high angle annular dark field (HAADF) images and analyses in Fig. 5 (note that the BF and HAADF images shown here covered most but not all of the distribution of particles). Under BF imaging, the noble metal particles appear dark, and, under HAADF imaging, they are bright. The noble metal phase particles found in the oxidized $\mathrm{Zr}$ layer ranged in size from a few 10 's to several 100's of nanometers, with the smaller particles appearing roughly spherical or ellipsoidal in shape and typically located at grain boundaries and vertices. Larger particles had greater variation in shape with some spherical but with some having much larger planar type shapes (Fig. 4). Size distributions of the particles with distance from the fuel-cladding interface was determined by measuring the diameter of each particle seen in STEM-HAADF images in 1- $\mu \mathrm{m}$ increments from the fuel. Within the first 3-4 $\mu \mathrm{m}$ from the fuel, the distribution was roughly bimodal. There were large particles with diameter greater than $100 \mathrm{~nm}$ coexisting with particles that were about $50-70 \mathrm{~nm}$ in diameter. As the depth into the Zr-oxide increased up to 5-6 $\mu \mathrm{m}$, the average size of the particles decreased although the number density increased. The number density of voids coincided with the bimodal nature of the particle size. These results matched fairly well with earlier reports of the concentration of fission products within the $\mathrm{FCl} .{ }^{41}$ The voids were correlated with the larger particles, and as will be shown later, these are most likely to be gas-filled bubbles that have been opened by the FIB-thinning process, resulting in the release of fission gas.

The STEM-HAADF images of a FIB-TEM lift-out specimen taken from the oxidized Zr layer are shown in Fig. 6a, along with STEMEDS point analyses from the oxidized Zr layer (b) and a noble metal phase particle (c). These EDS point analysis were obtained from the $\mathrm{ZrO}_{2}$ matrix and metal particle as indicated by the green and yellow crosses labeled in Fig. 6a, respectively. TEM-BF images of the metallic nanoparticle at increasing magnifications (the blue box outlines the magnified region) are shown in Fig. 6f with figures $(\mathrm{g})$ and $(\mathrm{h})$ the selected area diffraction at two different tilts determined on the analysis of the diffraction patterns. In agreement of the work by Buck et al. ${ }^{13}$ and Cui et al., ${ }^{9}$ STEM/ TEM investigations here show that the larger noble metal phase particles were not single crystals but instead were polycrystalline with grain sizes on the order of nanometers. Figure 6a shows a SEM image using a STEM detector of a large noble metal phase particle that shows that it is comprised of several crystals. The lattice fringe images also revealed stacking faults in the metallic phase.

The EDS spectra in Fig. $6 \mathrm{~b}, \mathrm{c}$ showed that the particles contained the $4 d$ metals and that there is at least some $U$ in solid solution within the Zr-oxide phase (additional EDS information is provided in the SI in Supplementary Fig. 4). The BF-TEM imaging and electron diffraction in Fig. $6 \mathrm{~d}-\mathrm{k}$ showed that the particles were polycrystalline with an $h c p$ structure that is characteristic of noble metal phase. The occurrence of the forbidden (001) reflection in this phase is due to the mix of $4 d$ elements in the structure.

In Fig. 7a, b, STEM-BF views of a different foil show a distribution of the metal particles. In many cases, the particles have a bimodal appearance with a void in the center. In Fig. 7c, a mixed elemental 
a
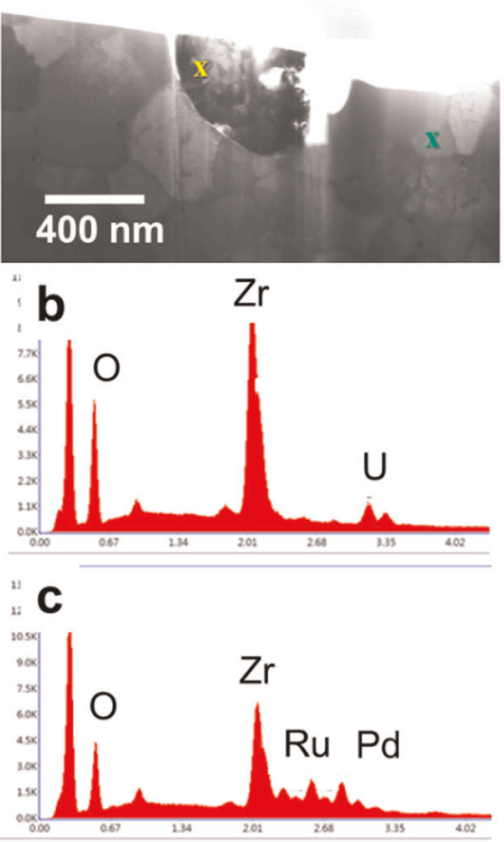
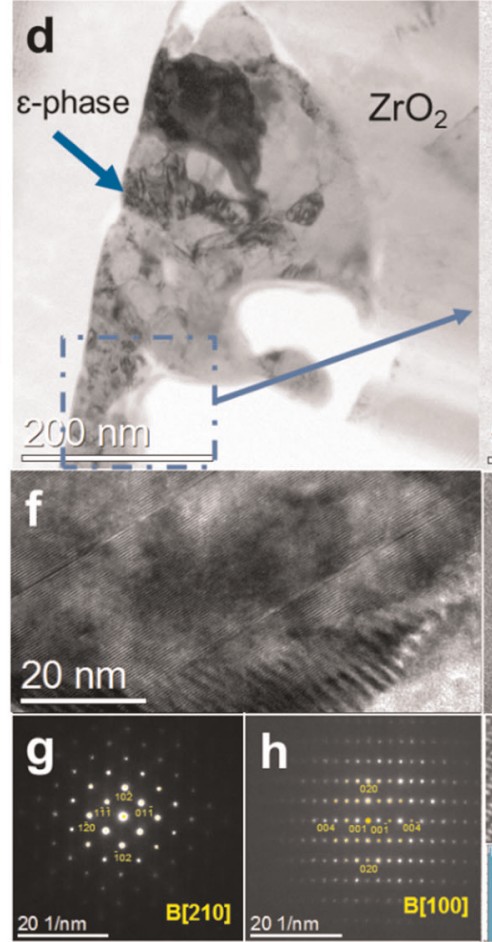

e
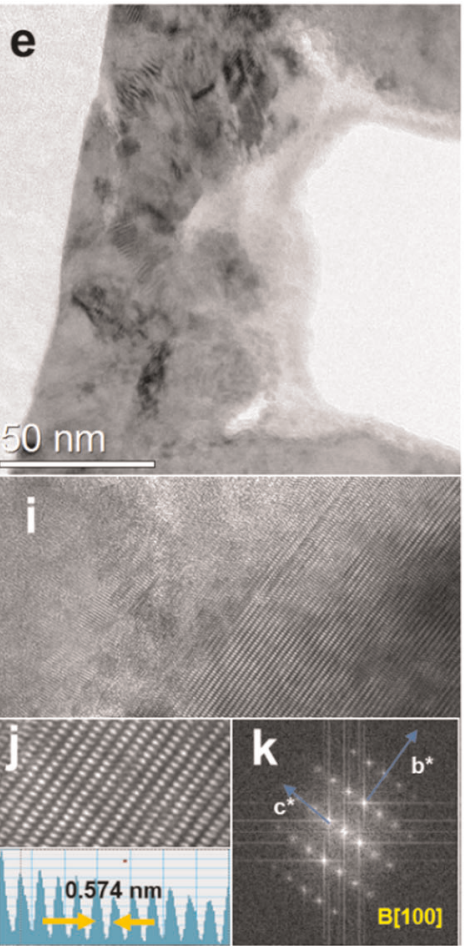

Fig. 6 Imaging and analysis of a large noble metal phase particle ( $\varepsilon$-phase) and surrounding liner. (a) HAADF-STEM image of the FIB-TEM specimen of the ATM-109 fuel-clad region. (b, c) STEM-EDS point analysis on the noble metal metal nanoparticle and crystalline cubic $\mathrm{ZrO}_{2}$ matrix indicated by a yellow and green cross labeled in (a), respectively. (d, e) BF-TEM images of the metallic nanoparticle at increasing magnifications (the blue box outlines the magnified region). (f) Magnified lattice image and ( $\mathbf{g}, \mathbf{h})$ selected area diffraction at two different tilts taken along $\mathrm{B}[210]$ and $\mathrm{B}[100]$. (i) Lattice fringe image showing stacking faults and enlarged image (j) displaying the $5.74 \AA$ corresponding to the (100) reflection in the metallic phase. The FFT of the lattice image is shown in (k) with the lattice directions $b^{*}[010]$ and $c^{*}[001]$ drawn on the image.
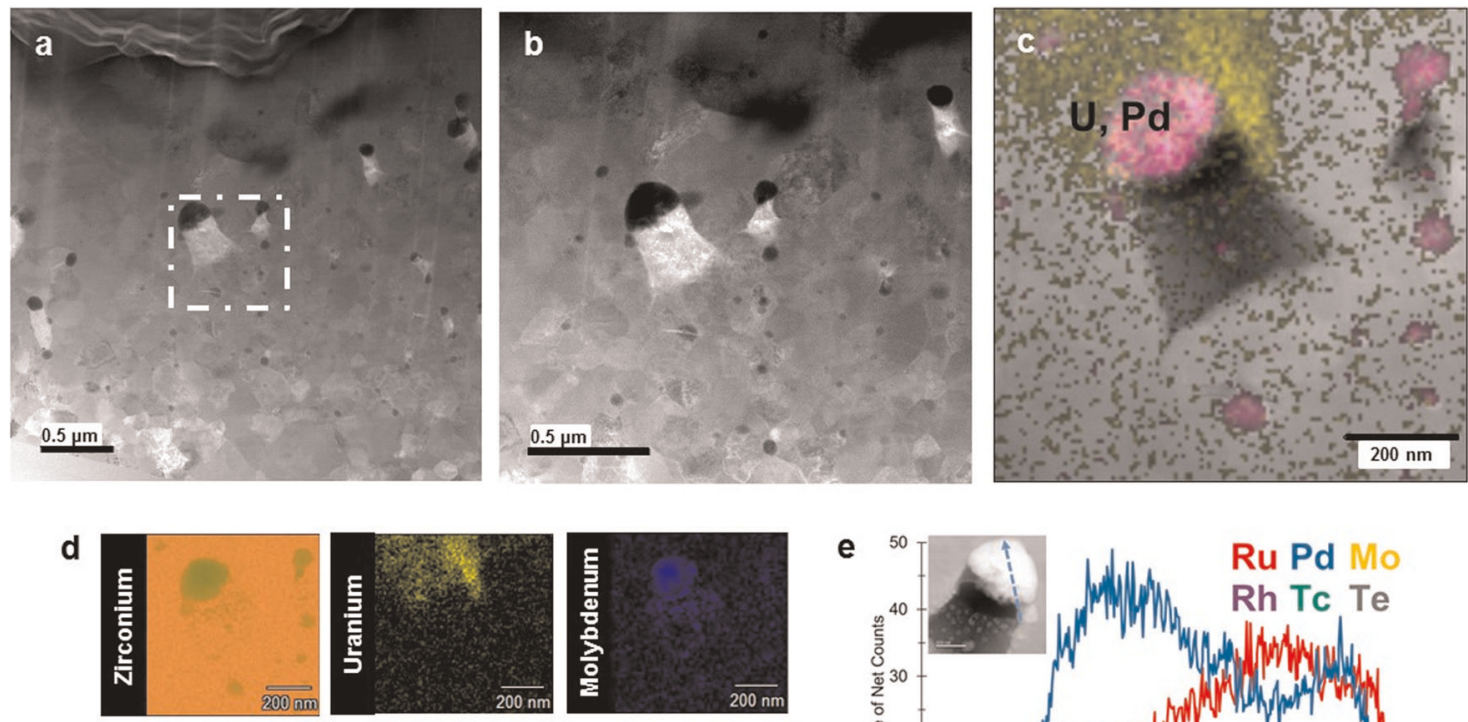

e
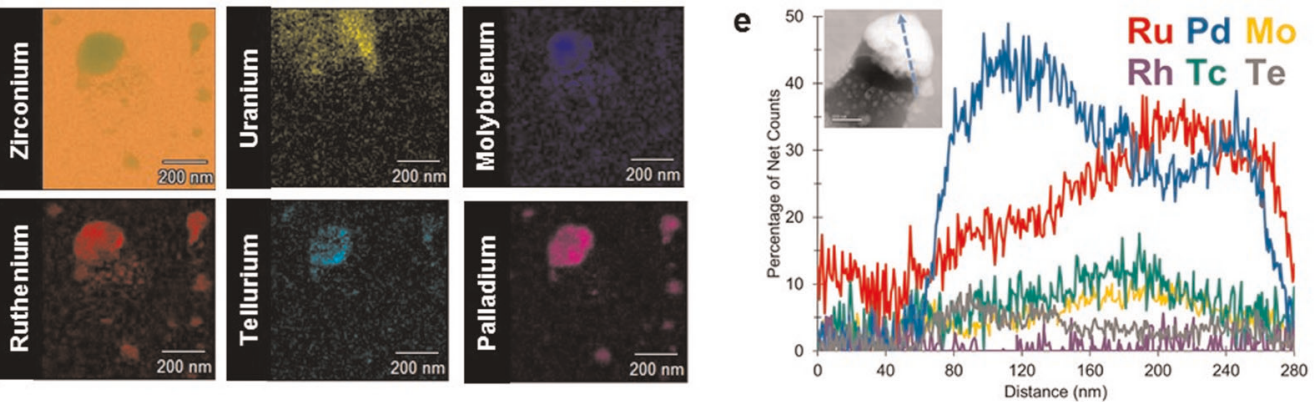

Fig. 7 Imaging and analysis of a thin foil lift-out. (a) low magnification BF view of foil, (b) higher magnification image of metal fissionproduct agglomerate, (c) mixed elemental maps and HAADF image of epsilon particle, (d) individual elemental maps of the particle, and (e) STEM-EDS line scan across the noble metal phase particle (from a different angle) showing chemical fractionation with Pd-Te overlapping with each other. 

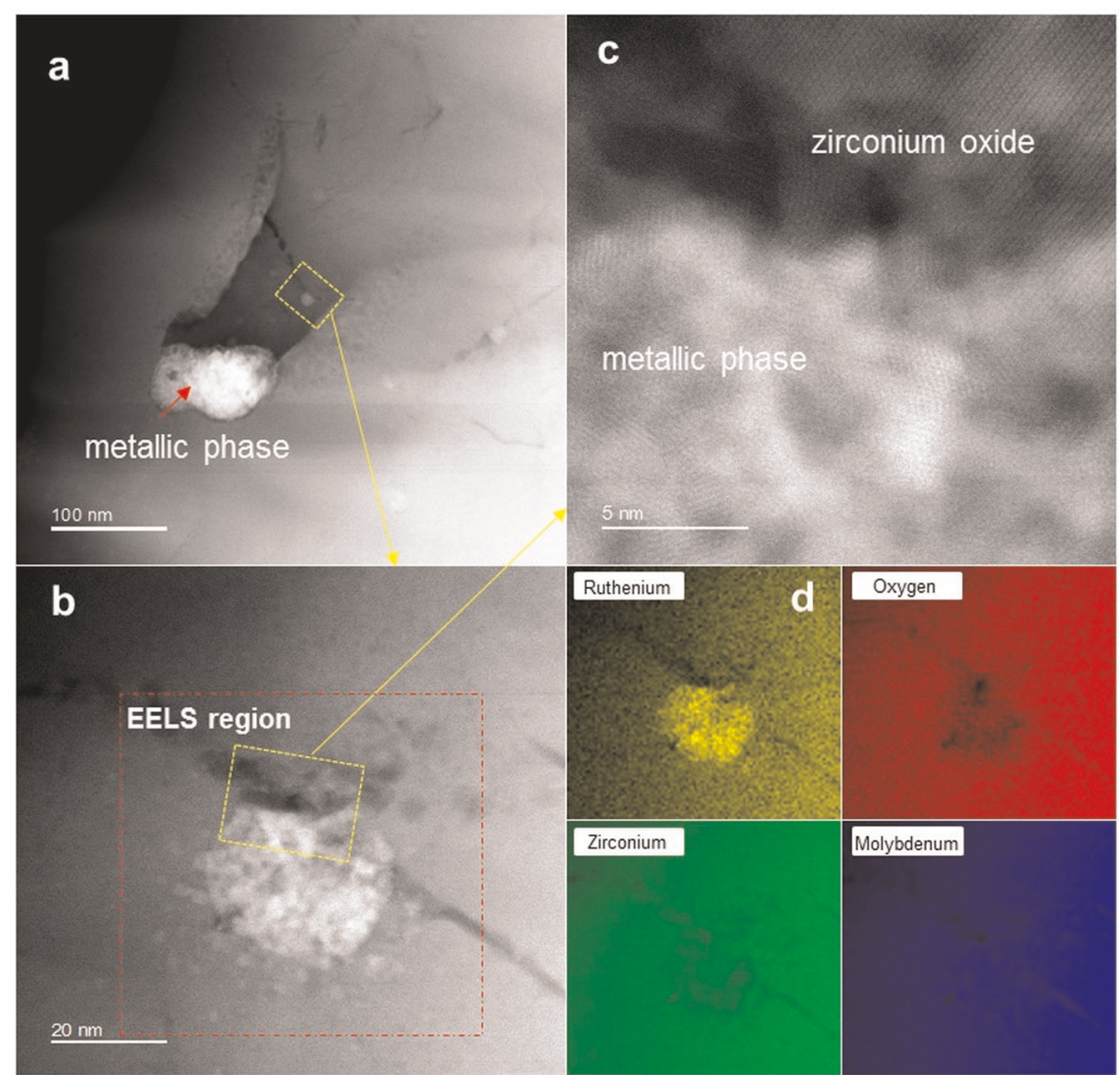

Fig. 8 Imaging and EELS elemental analysis of nanometer-sized metallic particles in the $\mathrm{ZrO}_{2}$ layer. (a) low magnification $\mathrm{HAADF}$ image, (b) higher magnification image of metal fission-product agglomerate, (c) atomic resolution image of the metallic phase, and (d) elemental maps using the Ru-L,O-K, Zr-L, and Mo-L edges.

map and STEM-HAADF image of a noble metal phase particle with the distribution of $U$ and $P d$ are shown. The elemental maps (see Fig. $7 d$ ) verified the expected $4 d$ elements (not all shown) and, in addition, the presence of significant quantities of Te. Tellurium was consistently associated with the noble metal phase particles, and elemental analysis of a dissolved portion of the phase showed quantities around $5 \%$ of the particle. ${ }^{36}$ These two studies (Pellegrini et al. and this study) provide clear association of Te with the noble metal phase particles that had not been previously established in earlier studies. A line-scan across the particle showed evidence of chemical fractionation. In these analyses, Pd and Te followed each other, and these two were anti-correlated with the other noble metal phase components, especially Ru (see Fig. 7e). Further analysis regarding the character and role of the Te association with noble metal phase particles is ongoing. Additional analysis of the noble metal phase particles is in the SI (Supplementary Figs 3 and 4).

To demonstrate the small size of the metallic particles, STEMEELS mapping was used to examine the microstructure. In Fig. 8, EELS mapping of one of the smaller metallic particles (on the order of $20 \mathrm{~nm}$ in diameter) gives evidence that, even at this size, the particles are agglomerates.

A gap (or possible gas bubble) was observed around many of the noble metal phase particles. To observe any gas associated with the particles, the gas could only be observed in regions where the sample was sufficiently thick to prevent its instant release during the preparation process. By using a higher energy electron beam ( $300 \mathrm{keV})$ equipped with dual EDS detectors, it was possible to examine the thicker locations in the foils. In Fig. 9d, a combination elemental map shows the occurrence of Xe gas located within a bubble and noble metal phase particles on the edges of the cavity. The EDS spectrum in Fig. 9b, confirms the presence of $\mathrm{Xe}$ in this region. As this was a thicker region of the specimen, it was not possible to obtain a reasonable EELS signal. This feature (Xe with noble metal phase particles) was seen repeatedly in regions with the particles/gaps still fully encapsulated within the sample matrix. A tomographic movie of the particles is available in the SI (Supplementary Videos 1 and 2). It shows the arrangement of the particles within the $\mathrm{Zr}$ oxide material.

\section{DISCUSSION}

Although the presence of fission products in cladding materials has been known to occur in $\mathrm{UO}_{2}$ spent fuels, the occurrence of discrete noble metal phase particles in the oxidized zirconium liner is the first direct evidence for these particles outside of the fuel matrix. This discovery begs several questions like, "How did these particles come to reside within the cladding? Did they form in situ or transport there whole?" While not yet fully understood, certainly the chemical mechanisms governing the occurrence, distribution, and concentration of noble metal phase particles in nuclear fuels are the result of a combination of radiation and thermal effects. It is unclear whether the chemical formation mechanisms would be dramatically different in the rim and cladding regions compared to the core of the fuel. Nonetheless, the observation of noble metal phase particles in cladding/ cladding liner is significant.

First, these particles likely could have formed in place through a combination of mechanisms that included penetration into the cladding by fission recoil of noble metals or their precursors, followed by radiation-enhanced diffusion (RED) and coalescence along grain boundaries. The $\mathrm{Zr}$ oxide phase at the $\mathrm{FCl}$ is found to be a thermodynamically high temperature stabilized phase 

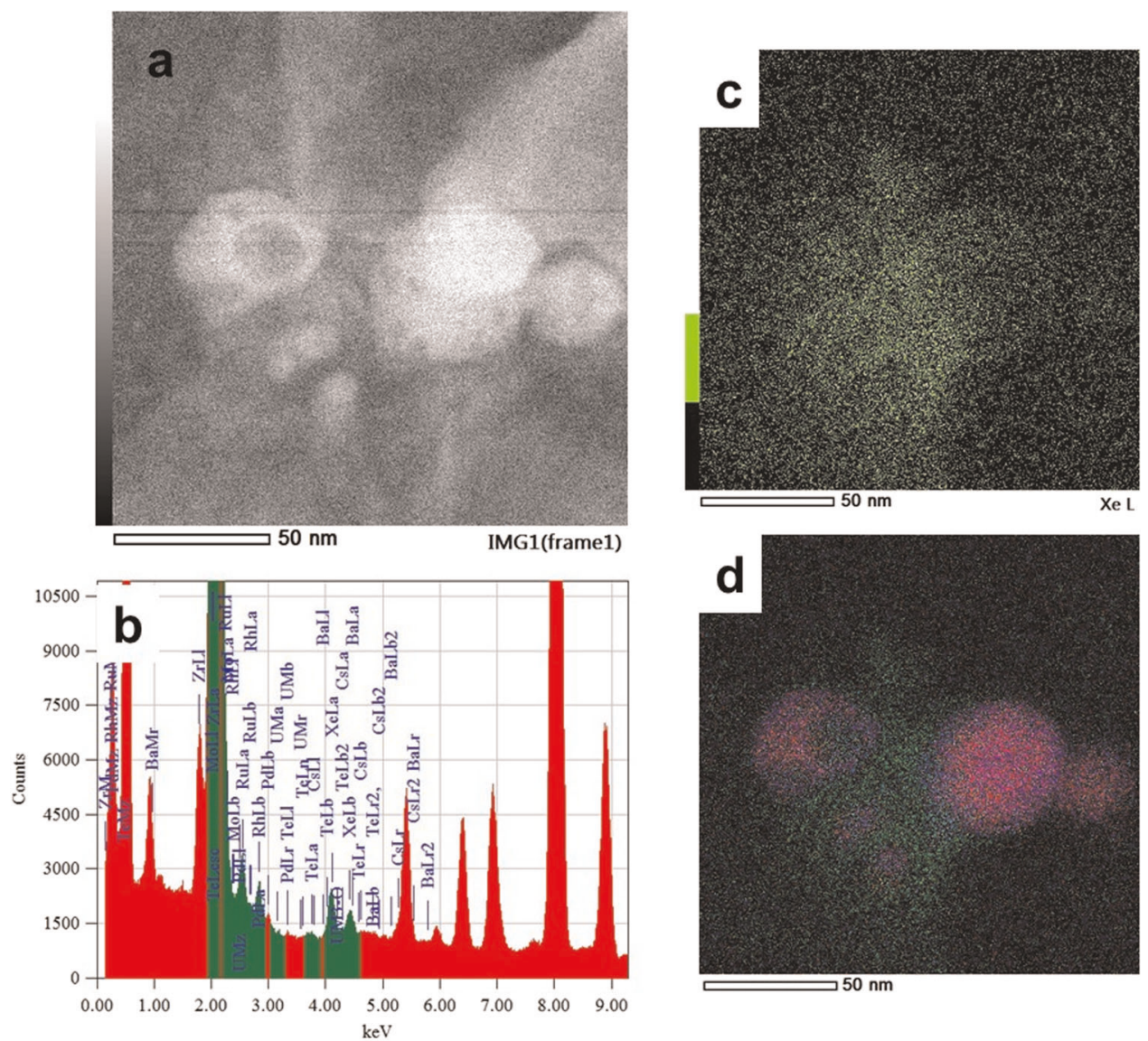

Fig. 9 Evidence for the presence of Xe associated with the noble metal phase particles. (a) HAADF image of noble metal phase particles within Zr cladding liner, (b) EDS analysis of region of interest confirming presence of $\mathrm{Xe}$, (c) EDS map of Xe, and (d) Combination Pd (red), $U$ (blue), and Xe (green) map showing Xe within gap associated with noble metal phase particles. In the SI, a tomographic view of similar precipitates shows the three-dimensional nature of the feature (Supplementary Videos 1 and 2).

(tetragonal phase $\left.\mathrm{ZrO}_{2}\right){ }^{40,42}$ The effect has been reported for high burn up fuel by Nogita and Une, ${ }^{42}$ and there it was suggested that high temperatures could be explained either by the thermal spike model $^{43,44}$ or a coulomb explosion model. ${ }^{45}$ For these models, neutron or heavy ion irradiation consequently provides the extensive local heating required to convert the more common monoclinic $\mathrm{ZrO}_{2}$ phase to the tetragonal phase. While the explanation fits a thermal argument, Lach et al., ${ }^{28}$ Ciszak et al., ${ }^{27}$ and others ${ }^{45,46}$ contend this change is better explained as the result of a fission recoil-radiation cascade defect structure, where point defect populations, particularly $\mathrm{O}$ vacancies, are stabilized due to the high dose rates. Neutron irradiation from the fuel alone does not produce the dose rate sufficient to overcome defect recombination and defect migration to sinks. Both explanations invoke disruptive processes that must be adopted to understand the evolution of the fuel matrix under fission recoil and neutron irradiation to a high burn up state. With this in mind, the size of the particles and number density of voids corresponds well with the grain size of the tetragonal $\mathrm{ZrO}_{2}$ which is largest nearest the fuel. ${ }^{28}$ The location of the particles predominantly at grain boundaries and grain triple junctions along with the likely large degree of RED highlights this as a probable mechanism for particle formation in the cladding liner oxide.

Looking at the characterization performed in this work, Fig. 9d appears to be a fractured cluster of metal phase particles around a Xe gas-filled void with uranium present in solid solution in the $\mathrm{ZrO}_{2}$. The Xe gas pressure is ostensibly the cause of the fracture. The pattern is repeated in multiple view graphs as in Fig. 7a-c, and the lower magnification in Fig. $4 a$, b. Here the images indicate the presence of voids to which metal particles have either gravitated to or have been propelled from. A by Schwantes et al. ${ }^{37}$ contends that a ballistic model well characterizes the behavior with respect to the Xe pressures required to overcome the yield strength of a $\mathrm{UO}_{2}$ void "cap".

Following this thought process, the presence of "jetting" features at the $\mathrm{FCl}$ and the uranium "cloud" and "tail" like those observable in in Figs 1 and 3 might suggest an alternative pathway into the cladding liner. Reduction of $\mathrm{UO}_{2}$ in contact with zirconium metal is frequently reported to reduce $\mathrm{UO}_{2}$ to $\mathrm{U}$ metal and other mixed $\mathrm{U}-\mathrm{Zr}-\mathrm{O}$ phases. ${ }^{40}$ In this study, the entire $\mathrm{ZrO}_{2}$ phase was tetragonal; ${ }^{28}$ therefore, any uranium in the $\mathrm{ZrO}_{2}$ is likely in solid solution. This uranium in solid solution and the noble metal phase particles may have been propelled from the fuel into the cladding liner. Such a violent mechanism, although unlikely or hard to imagine, becomes more probable with both high temperature and pressure gradients that appear to be in force during the later stages of the fuel's life. ${ }^{42}$ There is evidence that Xe bubble rupture within the cladding liner causes particles to break up and move $10 \mathrm{~s}$ or even $100 \mathrm{~s}$ of nanometers based on the large noble metal particle with a trail of open space with smaller particles in that space in Figs 4 and 7. However, the lack of recrystallized grains along the path from the $\mathrm{UO}_{2}$ to the noble metal phase particles prevents strongly embracing this mechanism. While pressure build up in the fuel due to fission gas production is not a new idea, discontinuous fracture of the fuel structure is, and again supports the notion that the evolution of fuel structure is a dynamical and sometimes violent process, and its explanation still requires new study and innovative modeling 
concepts. It is important to note that mechanisms for particle entry into the cladding liner (diffusion or eruption presented here) are not mutually exclusive.

This study has also confirmed the observation by Pellegrini et al. $^{36}$ that $\mathrm{Te}$ is a major fission product in these particles. The exact nature of the association of Te with the noble metal phase particles needs to be studied further. Though an association of Te with elements that comprise the noble metal phase has been hypothesized (particularly Pd) as possible, ${ }^{6}$ such phases have not been directly identified in light water reactor fuels. Here, we present a direct correlation within the noble metal phase particles of Te and Pd, observed in Fig. 7. Concurrent research also found a $\mathrm{Pd}$-Te phase. ${ }^{47-49}$ In that research, ${ }^{49}$ a separate $\mathrm{Pd}-\mathrm{Te}$ phase often associated with the noble metal phase particles in the fuel itself. In the work presented here, we found that when examining the noble metal phase particles themselves, there is a separate palladium telluride phase within the noble metal phase particles at the nano-scale (Fig. 7). Ongoing research will further detail this phase and its formation within these metal particles.

Xenon is the most common fission product generated in SNF; however, detection of it requires specimens sufficiently thick to prevent venting during sample preparation. Some of the first observations of $\mathrm{Xe}$ in $\mathrm{UO}_{2}$ spent fuels were made by Thomas et al. $^{50}$ where it was theorized that the Xe must be under significant pressure given the concentration observed. In this paper, we have made the first observations of xenon in a section of cladding. Locating Xe required using a higher energy $300 \mathrm{keV}$ beam coupled with multiple EDS detectors that enables exploration of thicker regions of the specimen. This Xe could be fission gas that forms pressurized bubbles just as the fission metal atoms form the noble metal phase particles in the cladding. The Xe gasfilled bubbles could also be generated in situ within the particle through decay of other elements in or around the metal particles (namely the newly associated Te within the particles). This may explain the observed separation of the metallic particles around the voids/bubbles in the $\mathrm{ZrO}_{2}$ matrix.

Although, the occurrence of fission products in the cladding and cladding oxide has been observed previously, this paper has documented a hitherto unknown complexity to the distribution of fission products and phase separation within fission product aggregates. Embedded fission products in cladding will impact the thermal properties of the fuel, as well as the ability to cleanly de-clad and re-process these materials. Answers to the questions of how the particles arrived in the cladding liner may have a significant impact on the characteristics of the fuel, thermal behavior, and the long-term disposal characteristics in the geologic repository. 3,24

\section{METHODS}

\section{Chemicals and materials}

As of July 2018, the ATM-109 fuel had been cooling for approximately 26 years. The material examined in this study included a fragment of cladding and a full-cross-section of the ATM-109 fuel. This work concentrated on examining the $\mathrm{FCl}$ zone. Future studies will report other aspects of the fuel condition.

\section{SEM Characterization}

Initial surface examinations using SEM-energy dispersive $x$-ray spectroscopy (EDS) were performed using an FEl (Hillsboro, OR, USA) Quanta 250FEG ${ }^{\text {TM }}$ field emission gun (FEG)-SEM. The SEM was used to locate regions of interest in the metal matrix microstructure. To minimize the effects of sample drift, a drift-correction mode was used during acquisition of the elemental maps.

\section{Preparation of TEM specimens}

Methods and precautions necessary to prepare lift-outs specimens from SNF using FIB-SEM have been described by Teague et al. ${ }^{10}$ and Aitkaliyeva et al. ${ }^{51}$ In this case, the SEM mounts containing the polished spent fuel fragments were mounted in specially designed metallic blocks that reduced the dose during handling. These blocks also contained insertion points for placing the lift-out row-holder. A FEl Helios $660 \mathrm{NanoLab}^{\mathrm{TM}}$ field emission gun (FEG) dual beam focused gallium ion beam/scanning electron microscope (FIB-SEM) equipped with an EDAX (EDAX Inc., Mahwah, NJ) compositional analysis system was used in the TEM foil preparation. The foils were on the order of $10 \times 10 \mu \mathrm{m}$ that resulted in sufficiently low radioactivity levels that they could be analyzed safely in the STEM/TEM. The current and accelerating voltage of the ion beam were adjusted from $9 \mathrm{pA}$ to $60 \mathrm{nA}$ and $2-30 \mathrm{kV}$, respectively, depending on the progress of the thinning operation. Each lift-out was attached to the OMNI $\mathrm{Cu}$ grid and further thinned by an ion beam operating at $2 \mathrm{kV}$ before removal from the sample chamber. Cross-sectional regions were obtained using a standard lift-out approach. The radioactive contamination state of the chamber was established before and after this process; however, some measurable fixed $\beta / \gamma$ activity particularly on the pole pieces did result from these processes.

\section{High resolution STEM characterization}

Specimens for transmission electron microscopy (TEM) were characterized in a JEOL (Japan) JEM ARM200C probe-corrected scanning transmission electron microscope (STEM) operated at $200 \mathrm{kV}$ and a JEOL ARM300F (GrandARM) probe-corrected STEM operated at $300 \mathrm{kV}$. Both tools were equipped with high angle annular dark field (HAADF) and bright field (BF) detectors and Bruker $x$-ray energy dispersive spectrometers (EDS). The GrandARM had dual EDS detectors providing a solid angle of $1.63 \mathrm{sr}$ and had Gatan Image Filter Quantum 663 electron energy-loss spectrometer. Diffraction patterns and electron micrographs were analyzed with Gatan Digital Micrograph ${ }^{\text {TM }} 3.0$.

\section{DATA AVAILABILITY}

Data that support the findings presented in this manuscript can be provided upon reasonable request by contacting the corresponding author.

Received: 22 March 2019; Accepted: 25 November 2019; Published online: 30 January 2020

\section{REFERENCES}

1. Cox, B. Pellet-clad interaction (PCI) failures of zirconium alloy fuel cladding-a review. J. Nucl. Mater. 172, 249-292 (1990).

2. Une, K., Imamura, M., Amaya, M. \& Korei, Y. Fuel oxidation and irradiation behaviors of defective BWR fuel rods. J. Nucl. Mater. 223, 40-50 (1995).

3. Ewing, R. C. Long-term storage of spent nuclear fuel. Nat. Mater. 14, 252-257 (2015).

4. Devanathan, R. et al. Modeling and simulation of nuclear fuel materials. Energ. Environ. Sci. 3, 1406-1426 (2010).

5. Wiss, T. et al. Recent results of microstructural characterization of irradiated light water reactor fuels using scanning and transmission electron microscopy. JOM 64, 1390-1395 (2012).

6. Kleykamp, H. The chemical state of the fission products in oxide fuels. J. Nucl. Mater. 131, 221-246 (1985).

7. Kleykamp, H., Paschoal, J. O., Pejsa, R. \& Thümmler, F. Composition and structure of fission product precipitates in irradiated oxide fuels: correlation with phase studies in the Mo- Ru-Rh-Pd and $\mathrm{BaO}-\mathrm{UO}_{2}-\mathrm{ZrO}_{2}-\mathrm{MoO}_{2}$ Systems. J. Nucl. Mater. 130, 426-433 (1985).

8. Bruno, J. \& Ewing, R. C. Spent nuclear fuel. Elements 2, 343-349 (2006).

9. Cui, D. et al. Characterization of alloy particles extracted from spent nuclear fuel. J. Nucl. Mater. 420, 328-333 (2012).

10. Teague, M., Gorman, B., King, J., Porter, D. \& Hayes, S. Microstructural characterization of high burn-up mixed oxide fast reactor fuel. J. Nucl. Mater. 441, 267-273 (2013).

11. Teague, M., Gorman, B., Miller, B. \& King, J. EBSD and TEM characterization of high burn-up mixed oxide fuel. J. Nucl. Mater. 444, 475-480 (2014).

12. Ray, I. L. F., Matzke, H., Thiele, H. A. \& Kinoshita, M. An electron microscopy study of the RIM structure of a $\mathrm{UO}_{2}$ fuel with a high burnup of $7.9 \%$ FIMA. J. Nucl. Mater. 245, 115-123 (1997).

13. Buck, E. C., Mausolf, E. J., McNamara, B. K., Soderquist, C. Z. \& Schwantes, J. M. Nanostructure of metallic particles in light water reactor used nuclear fuel. J. Nucl. Mater. 461, 236-243 (2015). 
14. Rest, J. et al. Fission gas release from $\cup_{2}$ nuclear fuel: a review. J. Nucl. Mater 513, 310-345 (2019).

15. Bradbury, B. T., Demant, J. T., Martin, P. M. \& Poole, D. M. Electron probe micro analysis of irradiated $\mathrm{UO}_{2}$. J. Nucl. Mater. 17, 227-236 (1965).

16. Bramman, J. I., Sharpe, R. M., Thom, D. \& Yates, G. Metallic fission-product inclusions in irradiated oxide fuels. J. Nucl. Mater. 25, 201-215 (1968).

17. Scheele, R., McNamara, B., Casella, A. M. \& Kozelisky, A. On the use of thermal NF as the fluorination and oxidation agent in treatment of used nuclear fuels. J. NuCl. Mater. 424, 224-236 (2012).

18. Utsunomiya, S. \& Ewing, R. C. The fate of the epsilon phase (Mo-Ru-Pd-Tc-Rh) in the $\mathrm{UO}_{2}$ of the Oklo natural fission reactors. Radiochim. Acta 94, 749-753 (2006).

19. Wronkiewicz, D. J., Watkins, C. S., Baughman, A. C., Miller, F. S. \& Wolf, S. F. Corrosion testing of a simulated five-metal epsilon phase in spent fuel. In Scientific Basis for Nuclear Waste Management XXV, Materials Res. Soc. Symp. Proc. (eds McGrail, B. P. \& Cragnolino, G. A.) 625-632 (Materials Research Scciety, Boston, MA, 2002).

20. Kaye, M. H., Lewis, B. J. \& Thompson, W. T. Thermodynamic treatment of noble metal fission products in nuclear fuel. J. Nucl. Mater. 366, 8-27 (2007).

21. Wronkiewicz, D. J. \& Buck, E. Uranium mineralogy and the geologic disposal of spent nuclear fuel. Rev. Mineral. Geochem. 38, 475-497 (1999).

22. McNamara, B., Hanson, B., Buck, E. \& Soderquist, C. Corrosion of commercial spent nuclear fuel. 2. Radiochemical analyses of metastudtite and leachates. Radiochim. Acta 93, 169 (2005)

23. Finn, P. A., Finch, R., Buck, E. \& Bates, J. Corrosion Mechanisms of Spent Fuel Under Oxidizing Conditions. MRS Proceedings Vol. 506 (1997).

24. Finn, P. A. et al. Colloidal products and actinide species in leachate from spent nuclear fuel. Radiochim. Acta 66, 189-196 (1994).

25. Buck, E. C., Finn, P. A. \& Bates, J. K. Electron energy-loss spectroscopy of anomalous plutonium behavior in nuclear waste materials. Micron 35, 235-243 (2004).

26. Piro, M. H. A. et al. in Reference Module in Materials Science and Materials Engineering (Elsevier, 2017)

27. Ciszak, C. et al. Micro-Raman analysis of the fuel-cladding interface in a high burnup PWR fuel rod. J. Nucl. Mater. 495, 392-404 (2017).

28. Lach, T. G. et al. Fission recoil-induced microstructural evolution of the fuelcladding interface $[\mathrm{FCl}]$ in high burnup BWR fuel. J. Nucl. Mater. 521, 120-125 (2019).

29. Hirabayashi, T. et al. Distributions of radionuclides on and in spent nuclear fuel claddings of pressurized water reactors. J. Nucl. Mater. 174, 45-52 (1990).

30. Vaidyanathan, S. et al. High burnup BWR fuel pellet performance. In Proc. of the Intl Topical Meeting on LWR Fuel Performance 471-477 (1997).

31. Wolf, S. F., Bowers, D. L. \& Cunnane, J. C. Analysis of high burnup spent nuclear fuel by ICP-MS. J. Radioanal. Nucl. Chem. 263, 581-586 (2005).

32. Kessler, S. H., Abrecht, D. G., Clark, R. A. \& Schwantes, J. M. Vibrational contributions to phase stability in the Mo-Ru system. J. Alloy. Compd. 689, 969-976 (2016).

33. Palomares, R. I. et al. Measuring the noble metal and iodine composition of extracted noble metal phase from spent nuclear fuel using instrumental neutron activation analysis. Appl. Radiat. Isot. 98, 66-70 (2015).

34. McNamara, B. K. et al. Separation of metallic residues from the dissolution of a high-burnup BWR fuel using nitrogen trifluoride. J. Fluor. Chem. 162, 1-8 (2014).

35. Buck, E. C., Mausolf, E. J., McNamara, B. K., Soderquist, C. Z. \& Schwantes, J. M. Sequestration of radioactive iodine in silver-palladium phases in commercial spent nuclear fuel. J. Nucl. Mater. 482, 229-235 (2016).

36. Pellegrini, K. L. et al. Chemical and isotopic characterization of noble metal phase from commercial UO 2 Fuel. Anal. Chem. 91, 6522-6529 (2019).

37. Schwantes, J. M. et al. Localized Fission Gas Production in Used Nuclear Fuel: Implications for Fission Gas Release, Cladding Corrosion and Next Generation of Fuel Design. PCCP. (in press).

38. Olander, D. R. The UO $\mathrm{U}_{2}$-Zircaloy chemical interaction. J. Nucl. Mater. 115, 271-285 (1983).

39. Walker, C. T., Bagger, C. \& Mogensen, M. Observations on the release of cesium from $\mathrm{UO}_{2}$ fuel. J. Nucl. Mater. 240, 32-42 (1996).

40. Dienst, W., Hofmann, P. \& Kerwin-Peck, D. K. Chemical Interactions Between $\mathrm{UO}_{2}$ and Zircaloy-4 from 1000 to $2000^{\circ} \mathrm{C}$. Nucl. Technol. 65, 109-124 (1984).

41. Lozano, N., Desgranges, L., Aymes, D. \& Niepce, J. C. High magnification SEM observations for two types of granularity in a high burnup PWR fuel rim. J. Nucl. Mater. 257, 78-87 (1998).

42. Nogita, K. \& Une, K. Formation of pellet-cladding bonding layer in high burnup BWR fuels. J. Nucl. Sci. Technol. 34, 679-686 (1997)

43. Nelson, R. S. An investigation of thermal spikes by studying the high energy sputtering of metals at elevated temperatures. Philos. Mag. 11, 291-302 (1965).

44. Kaoumi, D., Motta, A. T. \& Birtcher, R. C. A thermal spike model of grain growth under irradiation. J. Appl. Phys. 104, 073525 (2008).
45. Dunlop, A., Lesueur, D. \& Barbu, A. Evolution of microstructure resulting from high electronic excitation during swift heavy ion irradiations. J. Nucl. Mater. 205 426-437 (1993).

46. Zhang, Y. et al. Grain growth and phase stability of nanocrystalline cubic zirconia under ion irradiation. Phys. Rev. B 82, 184105 (2010).

47. Clark, R. A. et al. In Book of abstracts. Eleventh International Conference on Methods and Applications of Radioanalytical Chemistry (MARC XI) Log 396 (Kona, HI, 2018).

48. Kessler, S., Lach, T., Buck, E., Schwantes, J. \& Clark, R. In Abstracts of papers of the American Chemical Societry (American Chemical Soceity, Washintion, DC, 2018).

49. Parrish, R. J. et al. Characterization of solid fission products in $13.7 \%$ FIMA MOX fuel using electron microscopy techniques. J. Nucl. Mater. 524, 67-79 (2019).

50. Thomas, L. E., Beyer, C. E. \& Chariot, L. A. Microstructural analysis of LWR spent fuels at high burnup. J. Nucl. Mater. 188, 80-89 (1992).

51. Aitkaliyeva, A., Madden, J. W., Miller, B. D., Cole, J. I. \& Gan, J. Comparison of preparation techniques for nuclear materials for transmission electron microscopy (TEM). J. Nucl. Mater. 459, 241-246 (2015).

\section{ACKNOWLEDGEMENTS}

This work was funded by Pacific Northwest National Laboratory under Laboratory Directed Research and Development (LDRD) funds with support from the Nuclear Process Science Initiative (NPSI). Pacific Northwest National Laboratory is operated by Battelle Memorial Institute for the United States Department of Energy under contract DE-AC05-76RL0-1830. Most experiments were performed in the PNNL RPL Facility within the RPL Microscopy Quiet Suite, which is outfitted with a FEI Helios 660 FIM/SEM and a JEOL GrandARM300 Aberration Corrected (AC) STEM/TEM. Additional TEM/STEM measurements were also collected on a JEOL ARM200 AC-STEM/TEM located in 3410, part of the Physical Sciences Facility. All of these instruments are part of PNNL's Institutional Microscopy Tools.

\section{AUTHOR CONTRIBUTIONS}

R.A.C., M.A.C., T.G.L., E.C.B., and K.L.P. conducted the experimental studies, performed SEM-FIB, SEM, and analyzed the data. T.G.L., E.C.B., and M.A.C. conducted the STEM/ TEM experiments, B.K.M. and J.M.S. contributed to the discussion of the results. R.A.C T.G.L., and E.C.B. co-wrote the paper with B.K.M. providing input. R.A.C. and J.M.S. supervised the project. R.A.C. and M.A.C. are co-first authors for this work.

\section{COMPETING INTERESTS}

The authors declare no competing interests.

\section{ADDITIONAL INFORMATION}

Supplementary information is available for this paper at https://doi.org/10.1038/ s41529-019-0107-0.

Correspondence and requests for materials should be addressed to R.A.C.

Reprints and permission information is available at http://www.nature.com/ reprints

Publisher's note Springer Nature remains neutral with regard to jurisdictional claims in published maps and institutional affiliations.

Open Access This article is licensed under a Creative Commons Attribution 4.0 International License, which permits use, sharing, adaptation, distribution and reproduction in any medium or format, as long as you give appropriate credit to the original author(s) and the source, provide a link to the Creative Commons license, and indicate if changes were made. The images or other third party material in this article are included in the article's Creative Commons license, unless indicated otherwise in a credit line to the material. If material is not included in the article's Creative Commons license and your intended use is not permitted by statutory regulation or exceeds the permitted use, you will need to obtain permission directly from the copyright holder. To view a copy of this license, visit http://creativecommons. org/licenses/by/4.0/.

(c) The Author(s) 2020 